\title{
Efeito do uso de ultrassom no processo de ultrafiltração de polpa de cajá-manga
}

\author{
Effect of the use of ultrasound in the ultrafiltration process of cajá-manga pulp \\ Efecto del uso de ultrasonidos en el proceso de ultrafiltración de pulpa de cajá manga
}

Recebido: 13/03/2021 | Revisado: 20/03/2021 | Aceito: 23/03/2021 | Publicado: 01/04/2021

Grasiele Scaramal Madrona

ORCID: https://orcid.org/0000-0002-8837-8424

Universidade Estadual de Maringá, Brasil

E-mail: grasiele@yahoo.com

Carolina Moser Paraíso

ORCID: https://orcid.org/0000-0003-3309-2557

Universidade Estadual de Maringá, Brasil

E-mail: carolina.moser@ hotmail.com

Suelen Siqueira dos Santos

ORCID: https://orcid.org/0000-0003-4484-7425

Universidade Estadual de Maringá, Brasil

E-mail: suelensiqueira.eng@gmail.com

Ana Paula Stafussa

ORCID: https://orcid.org/0000-0002-7884-0195 Universidade Estadual de Maringá, Brasil E-mail: anastafussa@gmail.com

Miria Hespanhol Miranda Reis

ORCID: https://orcid.org/0000-0002-7513-907X

Universidade Federal de Uberlândia, Brasil

E-mail: miria@feq.ufu.br

\begin{abstract}
Resumo
O processo de filtração por membrana é uma alternativa promissora para a purificação de extratos ricos em compostos bioativos, mas algumas desvantagens devem ser consideradas, como o entupimento da membrana. Assim, o objetivo do presente estudo foi a avaliação da filtração por membrana assistida por ultrassom na purificação do extrato de cajámanga. O pré-tratamento com membrana de microfiltração $(0,8 \mu \mathrm{m})$ foi eficiente na clarificação do extrato sendo que ocorreu uma redução de $92 \%$ e $22 \%$ para turbidez e sólidos totais, respectivamente. A aplicação de ultrassom aumentou o fluxo em $64 \%$ para a membrana de $50 \mathrm{kDa}$ e apenas $16 \%$ para a de $150 \mathrm{kDa}$. Além disso, ocorreu uma melhora significativa do fluxo estabilizado para as filtrações com ultrassom, sendo os fluxos aumentaram em $35 \%$ para a membrana de $50 \mathrm{kDa}$ e $32 \%$ para a membrana de $150 \mathrm{kDa}$. O teor de sólidos totais após o processo de filtração por membrana assistido por ultrassom diminuiu em $20 \%$ e $7 \%$, para as membranas de $50 \mathrm{kDa}$ e $150 \mathrm{kDa}$, respectivamente. A microscopia eletrônica de varredura indicou que ocorreu maior aglomeração de partículas nas membranas no processo sem ultrassom após a permeação do extrato. Os resultados sugerem que o processo sequencial de pré-tratamento (microfiltração) e filtração através de membranas de ultrafiltração assistido por ultrassom pode ser potencialmente aplicado para produzir extratos clarificados de cajá-manga, com teores significativos de compostos bioativos e baixo entupimento no processo de filtração.
\end{abstract}

Palavras-chave: Fouling; Compostos bioativos; Membrana; S. dulcis Parkinson.

\begin{abstract}
The membrane filtration process is a promising alternative for the purification of extracts rich in bioactive compounds, but some drawbacks should be considered such as the membrane fouling. Thus, the aim of the present study was the evaluation of membrane filtration assisted by ultrasound in the purification of cajá-manga extract. Pretreatment with microfiltration membrane $(0.8 \mu \mathrm{m})$ was efficient in clarifying the extract, with a reduction of $92 \%$ and $22 \%$ for turbidity and total solids, respectively. The application of ultrasound increased the flow by $64 \%$ for the $50 \mathrm{kDa}$ membrane and only $16 \%$ for the $150 \mathrm{kDa}$ membrane. In addition, there was a significant improvement in the stabilized flow for ultrasound filtrations, with stabilized flows increasing by $35 \%$ for the $50 \mathrm{kDa}$ membrane and $32 \%$ for the $150 \mathrm{kDa}$ membrane. The total solids content after the ultrasound-assisted membrane filtration process decreased by $20 \%$ and $7 \%$, for the $50 \mathrm{kDa}$ and $150 \mathrm{kDa}$ membranes, respectively. The scanning electron microscopy indicated that there was a greater agglomeration of particles in the membranes in the process without ultrasound after the permeation of the extract. The results suggest that the sequential pre-treatment process (microfiltration) and filtration through ultrasound assisted ultrafiltration membranes can potentially be applied to produce clarified cajámanga extracts, with significant levels of bioactive compounds and low fouling in the filtration process.
\end{abstract}

Keywords: Fouling; Bioactive compounds; Membrane; S. dulcis Parkinson. 


\begin{abstract}
Resumen
El proceso de filtración por membranas es una alternativa prometedora para la purificación de extractos ricos en compuestos bioactivos, pero se deben considerar algunas desventajas, como la obstrucción de la membrana. Así, el objetivo del presente trabajo fue la evaluación de la filtración por membranas asistida por ultrasonido en la purificación del extracto de cajá manga. El pretratamiento con membrana de microfiltración $(0,8 \mu \mathrm{m})$ fue eficaz en la clarificación del extracto, con una reducción del $92 \%$ y $22 \%$ de turbidez y sólidos totales, respectivamente. La aplicación de ultrasonidos incrementó el flujo en un $64 \%$ para la membrana de $50 \mathrm{kDa}$ y solo un $16 \%$ para la membrana de $150 \mathrm{kDa}$. Además, hubo una mejora significativa en el flujo estabilizado para las filtraciones por ultrasonido, con flujos estabilizados aumentando en un $35 \%$ para la membrana de $50 \mathrm{kDa}$ y en un $32 \%$ para la membrana de $150 \mathrm{kDa}$. El contenido total de sólidos después del proceso de filtración por membrana asistido por ultrasonido disminuyó en un $20 \%$ y un $7 \%$, para las membranas de $50 \mathrm{kDa}$ y $150 \mathrm{kDa}$, respectivamente. La microscopía electrónica de barrido indicó que hubo una mayor aglomeración de partículas en las membranas en el proceso sin ultrasonidos luego de la permeación del extracto. Los resultados sugieren que el proceso de pretratamiento secuencial (microfiltración) y la filtración a través de membranas de ultrafiltración asistidas por ultrasonido pueden potencialmente aplicarse para producir extractos clarificados de anacardo y mango, con niveles significativos de compuestos bioactivos y baja obstrucción en el proceso de filtración.
\end{abstract}

Palabras clave: Fouling; Compuestos bioactivos; Membrana; S. dulcis Parkinson.

\title{
1. Introdução
}

S. dulcis Parkinson (Anacardiaceae), conhecida como cajá-manga, é originária de Polynesian Islands, sendo cultiva e comercializada no Brasil para o consumo in natura, fabricação de concentrados de frutas de alta qualidade, sorvetes, sucos, néctares e geleias, todos com alto valor nutricional e comercial (Louback, Pereira, De Souza, De Oliveira, \& Da Silva, 2016). Essa fruta apresenta elevado teor de compostos bioativos com caráter antioxidante, com 220,57 $\mu \mathrm{g} / 100 \mathrm{mg}$ de compostos fenólicos e 106,37 $\mu \mathrm{g} / \mathrm{g}$ de carotenoides (dos Santos, Rodrigues, Cardoso, Reis, \& Madrona, 2018). Devido à importância dos diferentes compostos bioativos presentes no cajá-manga, a obtenção dos extratos e purificação são de grande interesse para a indústria de alimentos e farmacêutica (Sousa, Cabral, Madrona, Cardoso, \& Reis, 2016).

O processo de filtração por membrana é uma técnica baseada na permeação de moléculas de soluto através de uma membrana semipermeável. Processos que utilizam membranas é uma alternativa eficiente para o tratamento de extratos e sucos de frutas, devido à preservação das características nutricionais e sensoriais do produto. Este processo é efetuado em temperatura ambiente e com o um mínimo de demanda energética, o que se mostra bastante interessante já que existem compostos termo sensíveis no produto (Bindes, Cardoso, Reis, \& Boffito, 2019; Madrona et al., 2018). Diversos trabalhos têm reportado a eficácia desse método no processamento de extratos vegetais, como hibisco (Paraíso et al., 2020) e camu-camu (Rodrigues et al., 2020).

Uma das desvantagens no processo de separação por membrana é a ocorrência de incrustações, o que leva a uma perda significativa no fluxo do permeado. Uma das estratégias que pode ser utilizada para reduzir a incrustação na membrana durante o processamento de extratos e sucos de frutas é a filtração por membrana assistida por ultrassom (Córdova, AstudilloCastro, Ruby-Figueroa, Valencia, \& Soto, 2020). Estudos recentes com filtração de chá verde e extrato de jenipapo observaram que a aplicação de energia ultrassônica nos processos de membrana tem a capacidade quebrar a torta formada na superfície da membrana, melhorando assim o desempenho durante a filtração (Madrona et al., 2018; Sousa et al., 2016).

Assim, o objetivo do presente estudo foi a avaliação da filtração por membrana assistida por ultrassom na purificação do extrato de cajá-manga.

\section{Metodologia}

O método científico utilizado nessa pesquisa foi um estudo de natureza quantitativa e experimental por se tratar da coleta e análise de dados numéricos. (Pereira, Shitsuka, Pereira, \& Shitsuka, 2018). 


\subsection{Extrato de cajá-manga}

As frutas foram adquiridas no Ceasa da cidade de Uberlândia-MG em setembro/2016, sendo todas de um mesmo lote. Após prévia seleção e higienização (hipoclorito a 100 ppm) separou-se manualmente a polpa do caroço, sendo que a polpa juntamente com a casca foi triturada em um mixer e analisada quanto ao $\mathrm{pH}$, acidez, cor, umidade e cinzas.

O extrato foi preparado adicionando-se água na proporção de 1:3 (m/m) e a extração de compostos bioativos ocorreu em chapa de aquecimento com agitação $(400 \mathrm{rpm})$ durante $30 \mathrm{~min}$ e temperatura de $75{ }^{\circ} \mathrm{C}$, sendo que a temperatura foi controlada por um controlador acoplado a chapa (Madrona et al., 2018). O extrato proveniente foi filtrado em filtro de café e armazenado no escuro a $25^{\circ} \mathrm{C}$. Após atingir a temperatura ambiente o extrato foi colocado em um reator do tipo batelada para que fosse iniciada a microfiltração.

\subsection{Processo de filtração por membrana}

\subsubsection{Microfiltração}

A microfiltração foi usada como um pré-tratamento para a remoção dos sólidos em suspensão e clarificação do extrato. $\mathrm{O}$ extrato recém-preparado e resfriado $\left(25^{\circ} \mathrm{C}\right)$ foi então microfiltrado em um reator batelada do tipo "dead end" (Figura 1). A membrana utilizada no processo era de éster de celulose (Millipore) com porosidade de $0,8 \mu \mathrm{m}$, área de filtração de $6,36 \times 10^{-3} \mathrm{~m}^{2}$ a uma pressão de 0,5 bar garantida por um manômetro do tipo bourdon conectado a um cilindro de nitrogênio (Silva, Rossi, Cardoso, \& Reis, 2016).

Figura 1. Esquema do módulo de microfiltração "dead end".

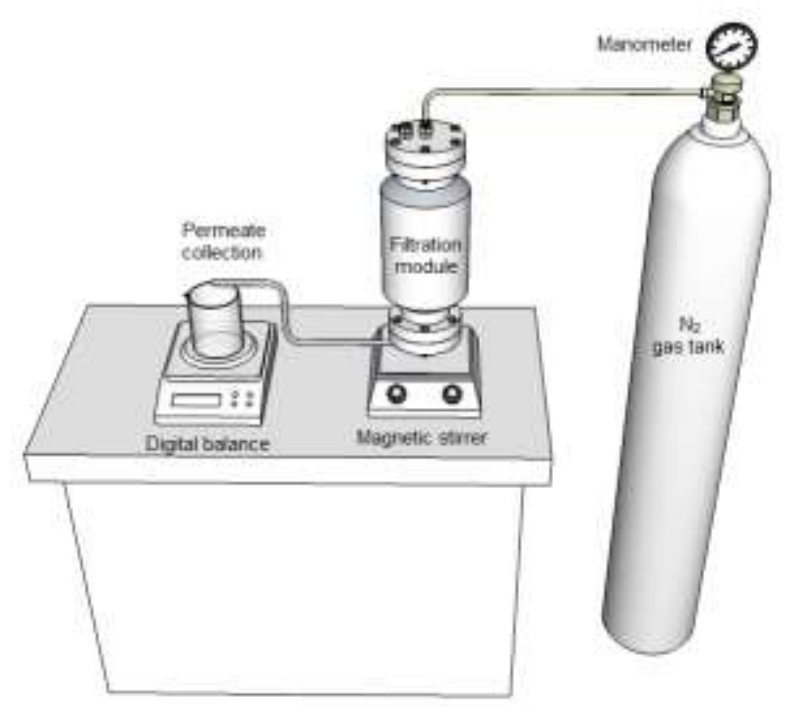

Fonte: Silva et al., (2016).

\subsubsection{Ultrafiltração com e sem ultrassom}

A Figura 2 apresenta um esquema do sistema de filtração utilizado para ultrafiltração onde pode-se operar em regime contínuo com membrana plana, em operação perpendicular ou tangencial. O sistema foi fabricado pela empresa Convergence (Holanda) e dispõe de sistema automático para controle e registro da pressão ao longo da filtração. 
Figura 2. Sistema de filtração contínuo.

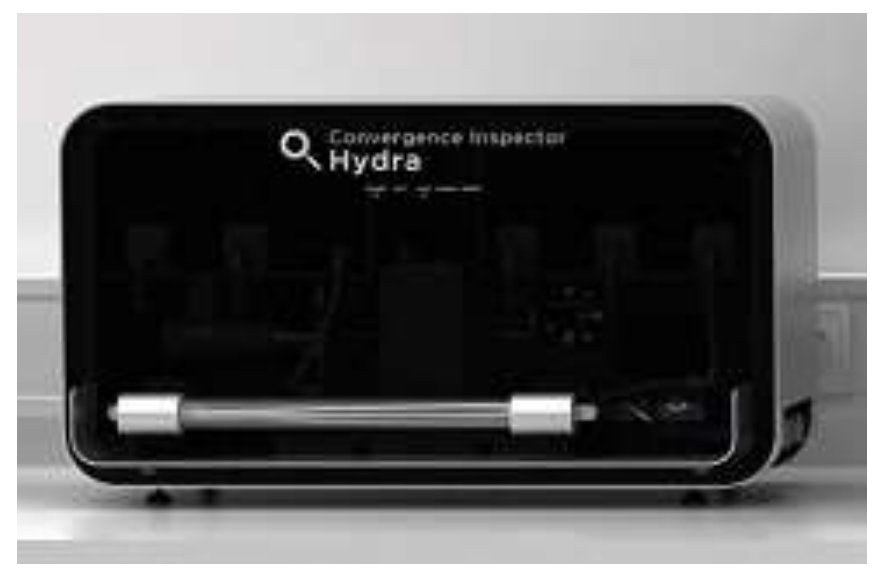

Fonte: Autores.

A área de permeação era de $3.84 \times 10^{-3} \mathrm{~m}^{2}$, utilizou-se pressão de 5 bar e membranas de 50 e $150 \mathrm{kDa}$ (Nadir), o processo foi realizado também utilizando banho ultrassônico. Para isso, o módulo com a membrana foi inserido dentro do aparelho de ultrassom (Ultracleaner 1650 Unique, freqüência de $40 \mathrm{KHz}$ ), e o mesmo foi ligado a partir do momento em que a filtração foi iniciada. Sabe-se que na filtração quando o módulo da membrana é inserido em um banho ultrassônico, o fouling sobre a membrana é reduzido aumentando assim o fluxo de permeado (Hou et al., 2015).

A vazão foi monitorada durante o processo. O processo foi interrompido a partir do momento em que a vazão do processo se estabilizasse.

Após iniciado o processo de filtração, amostras foram coletadas durante todo o processo para medir o comportamento do fluxo em função do tempo de filtração. O fluxo foi calculado utilizando a Equação 1.

$$
I=\frac{m}{A t}
$$

onde $J$ é o fluxo de permeado, $\left(\mathrm{Kg} \mathrm{m}^{-2} \mathrm{~h}^{-1}\right), m$ é a massa do permeado $(\mathrm{Kg})$ e $t$ é o intervalo de tempo para coletar a amostra do permeado (h).

Foram realizados quatro tratamentos sendo eles: $50 \mathrm{kDa}$ com ultrassom, $50 \mathrm{kDa}$ sem ultrassom, $150 \mathrm{kDa}$ com ultrassom e $150 \mathrm{kDa}$ sem ultrassom.

\subsection{Microscopia eletrônica de varredura}

O estudo de imagem das membranas realizado por microscopia eletrônica de varredura (MEV), utilizando microscópio eletrônico de varredura JEOL modelo JSM-6060 LV. As amostras (membranas usadas) foram fixadas em um suporte metálico com auxílio de uma fita dupla-face de carbono e recobertas com uma fina camada de ouro. A visualização foi realizada em aumentos de 100 a 3000 vezes, com uma voltagem de excitação de 15 kV (Machado, Mello, \& Hubinger, 2015).

\subsection{Análises físico-químicas dos extratos}

Os compostos fenólicos totais foram determinados pelo método de Folin Ciocalteau. A análise da absorbância foi realizada em espectrofotômetro UVmini - 1240 Shimadzu a uma frequência de $725 \mathrm{~nm}$, sendo realizadas em triplicata. Os resultados foram expressos em miligrama de ácido gálico equivalente por grama de amostra (mgEAG/g) (Singleton \& Rossi, 1965). A cor foi avaliada por meio do colorímetro portátil Minolta® CR400, utilizando sistema CIEL*a*b*, onde foram medidas as coordenadas: L, representando a luminosidade em uma escala de 0 (preto) a 100 (branco); a* que representa uma escala de tonalidade variando de vermelho $(0+a)$ a verde $(0-a)$ e $b^{*}$ que representa uma escala de amarelo $(0+b)$ a azul $(0$ - 
b). Todas as determinações foram feitas em triplicata. A turbidez foi medida utilizando turbidimetro (Tecnopon TB-1000) em unidades nefelométricas de turbidez (NTU). Os sólidos totais, $\mathrm{pH}$, acidez, umidade e cinzas foram analisados segundo AOAC (2016), e o brix foi medido utilizando refratômetro (HI 96801 Hanna), todas as análises foram realizadas em triplicata.

A polpa da fruta cajá-maga foi caracterizada para determinar as concentrações de ácido gálico, ácido caféico, ácido clorogênico, rutina e quercitina usando cromatografia líquida em um HPLC Shimatzu (modelo LC-20A Prominence) equipado com uma coluna Discovery HS C18. Análises cromatográficas foram realizados a $280 \mathrm{~nm}$ e $40^{\circ} \mathrm{C}$. Uma amostra de $10 \mu \mathrm{L}$ foi injetado automaticamente a uma taxa de fluxo de $0,7 \mathrm{~mL}$ min-1. A fase móvel consistia em $2 \%$ (v/v) de ácido acético em água (eluente A) e 0,5\% de ácido acético em água e acetonitrila $(50: 50 \mathrm{v} / \mathrm{v}$, eluente B) usando um programa gradiente (Ribeiro et al., 2015).

Os dados foram analisados no Sisvar 5.3 por Anova e teste de Tukey $(\mathrm{p}<0,05)$ (Ferreira, 2008).

\section{Resultados e Discussão}

\subsection{Caracterização da fruta in natura}

A Tabela 1 apresenta as características físico-químicas da polpa in natura de cajá-manga em termos de pH, acidez, umidade, cinzas e cor instrumental. Analisando pH e acidez os valores diferiram em relação ao estudo de Breda et al. (2013) que encontraram valores de 3,32 e 0,50 para a polpa da fruta, respectivamente. O cajá-manga é uma fruta muito apreciada pelo seu sabor típico, que varia de ácido a doce, dependendo da maturação do fruto, das condições climáticas e a colheita (dos Santos et al., 2018).

Em relação aos valores de umidade e cinzas, os valores estão próximos aos encontrados na literatura de $82,71 \%$ e 0,39\%, respectivamente (Breda et al., 2013; Lago-Vanzela et al., 2011). Analisando os parâmetros da cor instrumental da polpa, os valores estão de acordo com os reportados pela literatura, próximos a 43,09 (L), -3,86 (a*) e 23,49 (b*) (dos Santos et al., 2018).

Tabela 1. Composição da polpa de cajá-manga.

\begin{tabular}{cc}
\hline Parâmetro & Polpa \\
\hline pH & $2,58 \pm 0,69$ \\
Acidez $(\%)$ & $1,46 \pm 0,07$ \\
Umidade (\%) & $87,33 \pm 0,03$ \\
Cinzas (\%) & $0,30 \pm 0,06$ \\
$\mathrm{~L}$ & $42,09 \pm 0,02$ \\
$\mathrm{a}^{*}$ & $-3,60 \pm 0,02$ \\
$\mathrm{~b}^{*}$ & $23,41 \pm 0,04$ \\
\hline
\end{tabular}

Fonte: Autores.

\subsection{Processo de filtração por membrana}

Considerando que pouca diferença se obteve utilizando um pré-tratamento antes da microfiltração (Madrona et al., 2018), o processo sequencial utilizado aqui considerou a microfiltração sem pré-tratamento seguida de ultrafiltração. A Figura 3 apresenta o fluxo do permeado em função do tempo para as filtrações do extrato com ultrassom e sem ultrassom de cajá manga através das membranas de 50 e $150 \mathrm{kDa}$ em 5 bar de pressão transmembrana. Observou-se uma queda brusca do fluxo nos primeiros 30 min da filtração, tal fato pode ocorrer, pois o peso molecular de alguns componentes do extrato é maior que $50 \mathrm{kDa}$, por isso a torta forma-se quase que instantaneamente, o que resulta em um baixo fluxo inicial destas membranas (Rao et al., 2011).

Os fluxos iniciais foram de 120,197, 256, $299 \mathrm{~L} / \mathrm{hm}^{2}$ para a membrana de $50 \mathrm{kDa}$ sem ultrassom, $50 \mathrm{kDa}$ com 
ultrassom, $150 \mathrm{kDa}$ sem ultrassom e $150 \mathrm{kDa}$ com ultrassom, respectivamente. Sendo que a aplicação de ultrassom aumentou o fluxo em $64 \%$ para a membrana de $50 \mathrm{kDa}$ e apenas $16 \%$ para a de $150 \mathrm{kDa}$. Além disso, notou-se uma melhora significativa do fluxo estabilizado para as filtrações com ultrassom, sendo os fluxos estabilizados foram de 4,5, 7,0, 14,2, 21,1 L/hm² para a membrana de $50 \mathrm{kDa}$ sem ultrassom, $50 \mathrm{kDa}$ com ultrassom, $150 \mathrm{kDa}$ sem ultrassom e $150 \mathrm{kDa}$ com ultrassom, respectivamente. $\mathrm{O}$ efeito do ultrassom na filtração por membrana de extrato de cajá manga não é relatado na literatura, mas o aumento do fluxo devido à aplicação do ultrassom já foi observado para outros fluídos. Estudo recente avaliou a aplicação do processo de ultrafiltração assistida por ultrassom para a purificação de compostos fenólicos do extrato de chá verde. A comparação entre os processos com e sem ultrassom mostraram que o fluxo em estado estável através da membrana de $5 \mathrm{kDa}$ foi 4 vezes maior no processo assistido por ultrassom do que no processo sem ultrassom (Sousa, Cabral, Madrona, Cardoso, \& Reis, 2016b). Sabe-se que o ultrassom tem a capacidade de transmitir quantidades substanciais de energia por meio de movimentos mecânicos e aumentando fenômeno de transferência de massa, levando uma redução do tempo de processamento. A aplicação de energia ultrassônica nos processos de filtração por membrana pode aumentar o fluxo ao quebrar a camada de torta na superfície da membrana, melhorando assim o desempenho durante a filtração (Córdova et al., 2020).

Figura 3. Fluxo em função do tempo obtidos para ultrafiltração de cajá-manga.

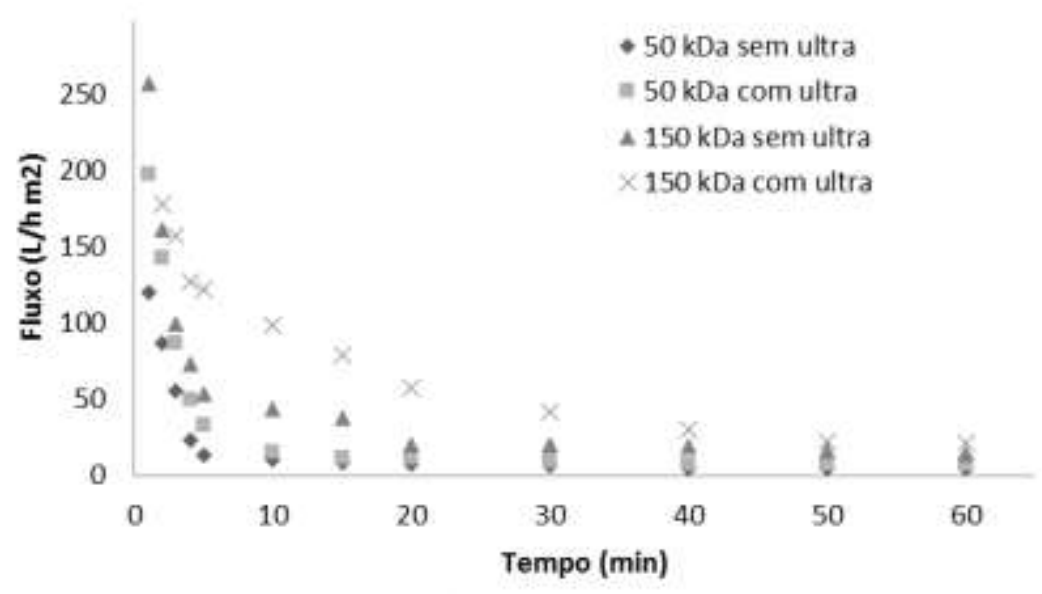

Fonte: Autores.

\subsection{Microscopia eletrônica de varredura (MEV)}

A Figura 4 apresenta a imagem da superfície das membranas utilizadas na presente pesquisa, antes e após a filtração. Pode-se observar nas micrografias que as membranas virgens possuem superfície lisa, ou seja, sem depósito de matéria orgânica, enquanto as membranas após o processo apresentam as superfícies heterogêneas, provavelmente devido à deposição de macropartículas na superfície das membranas durante a filtração. Tylkowski et al. (2010) observaram depósito de partículas na superfície da membrana após a nanofiltração de extrato de própolis e relataram que esta deposição poderia ser atribuída a macronutrientes ou a ceras, lipídios, óleos essenciais, pólen e minerais extraídos da própolis.

Em relação à superfície das membranas após a filtração do extrato nota-se que ocorre maior aglomeração de partículas no processo sem ultrassom, ao que parece tal processo pode fracionar as partículas facilitando assim sua passagem pela membrana consequentemente aumentando o fluxo de permeado (Figura 3). 
Figura 4. Microscopia eletrônica de varredura da superfície das membranas: (a) membrana 50 kDa antes da filtração; (b) membrana $150 \mathrm{kDa}$ antes da filtração; (c) membrana $50 \mathrm{kDa}$ após a filtração sem ultrassom; (d) membrana 150 kDa após a filtração sem ultrassom; (e) membrana $50 \mathrm{kDa}$ após a filtração com ultrassom; (f) membrana 50 kDa após a filltração com ultrassom.
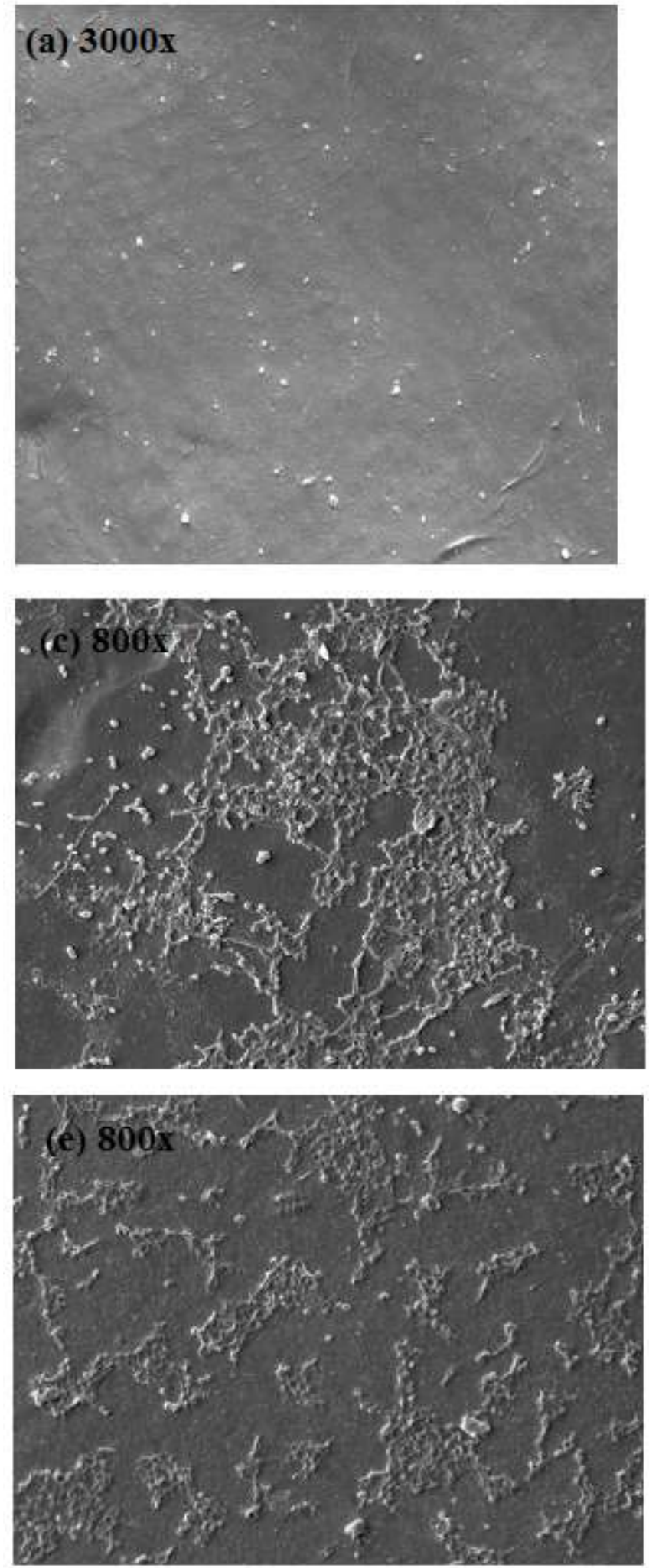
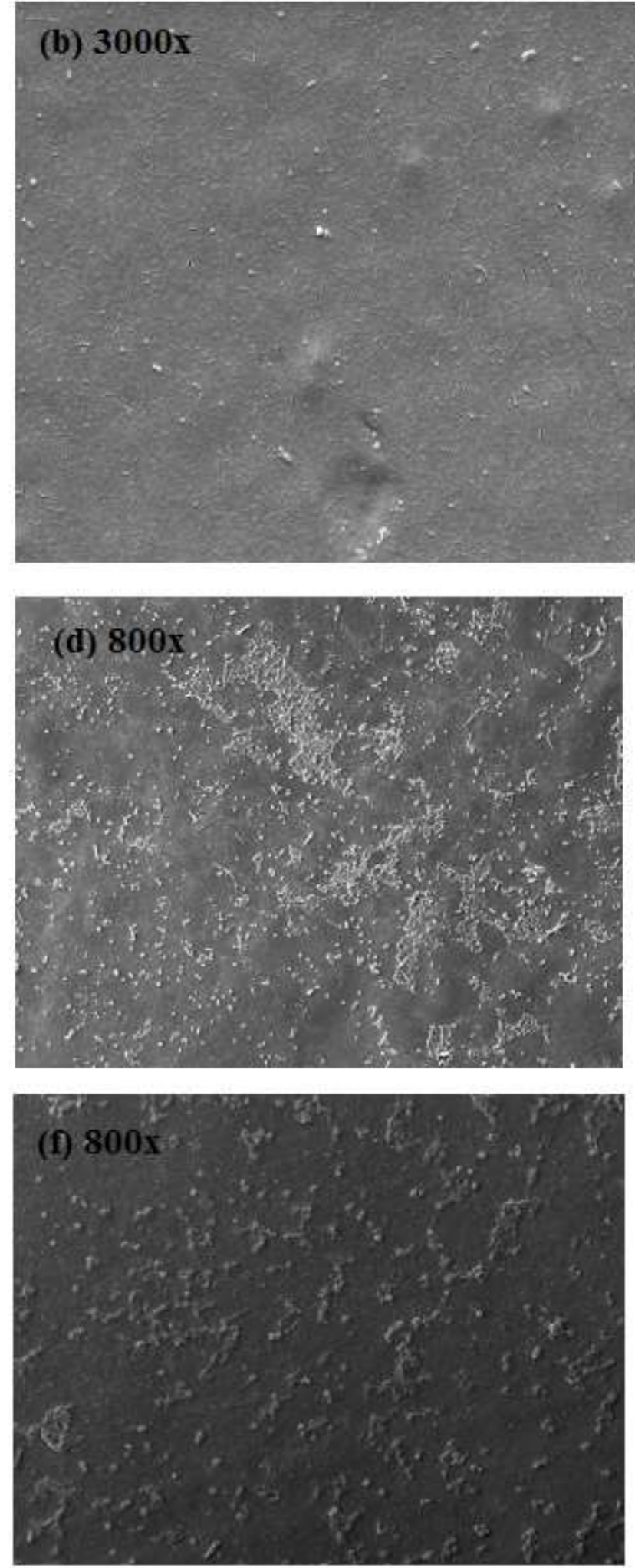

Fonte: Autores. 


\subsection{Análises físico-químicas dos extratos}

A Tabela 2 apresenta os dados obtidos para as amostras de extrato, permeado da microfiltração, permeado e concentrado de cajá-manga no processo de ultrafiltração com ultrassom, considerando que os melhores valores de fluxo foram obtidos nesta condição.

Observou-se que após o pré-tratamento com a membrana de microfiltração ocorreu uma redução de $92 \%$ e $22 \%$ para turbidez e sólidos totais, respectivamente. Além disso, ocorreu a clarificação do extrato, sendo que a luminosidade do extrato aumentou significativamente de 65,7 para 85,9. Em relação aos compostos bioativos, observou-se que ocorreu um aumento no teor de ácido gálico e ácido cafeíco, e uma retenção significativa no teor da rutina. Estudo recente que avaliou a membrana de microfiltração com poro de $0,8 \mu \mathrm{m}$ no tratamento de extrato de chá verde observou a diminuição da turbidez do extrato e a concentração de sólidos, enquanto a intensidade da cor e a concentração total de polifenóis permaneceu (Sousa et al., 2016).

Observou-se que a maioria dos resultados são significativamente diferentes $(p \leq 0,05)$ após a permeação do extrato de alimentação na membrana de $50 \mathrm{kDa}$ e $150 \mathrm{kDa}$. Ocorreu uma retenção de 15,6\% de ácido gálico, 24\% de ácido cafeíco e $63,70 \%$ de compostos fenólicos para a membrana de $50 \mathrm{kDa}$. E para a membrana de $150 \mathrm{kDa}$ ocorreu uma retenção de $42,13 \%$ de ácido gálico, 47,9 \% de ácido cafeíco, $85 \%$ de ácido clorogênico, 38\% de quercetina e 32\% de compostos fenólicos. A retenção de compostos bioativos não é desejável em um processo de clarificação, a fim de manter as propriedades funcionais do extrato, porém pequenas perdas são comuns após o processo de filtração por membranas (Bindes et al., 2019). A retenção desses compostos pode ser atribuída à interação com outros compostos específicos dos extratos, bem como com os compostos que contribuem com fenômenos incrustantes na superfície ou nos poros da membrana (Meija, Parpinello, Versari, Conidi, \& Cassano, 2019). Estudo semelhante sobre clarificação de jenipapo a partir da filtração através membrana assimétrica de cerâmica com 1 bar de pressão transmembrana observou reduções das concentrações de compostos fenólicos totais em 17 \% (Terra, Madrona, Ferreira, Cardoso, \& Reis, 2019).

Além disso, notou-se que a partir do extrato de alimentação ocorreu uma concentração do composto quercetina de 28,91 para 40,12 mg/mL após a permeação na membrana de $150 \mathrm{kDa}$. O mecanismo de separação no processo de ultrafiltração é baseado principalmente em um efeito de peneiramento e as partículas são separadas de acordo com suas dimensões, embora outros fatores, como forma e carga, bem como as interações entre a própria membrana e as partículas sendo filtradas, desempenhem um papel fundamental (Cassano, De Luca, Conidi, \& Drioli, 2017). Estudo anterior avaliou o potencial de membranas planas de ultrafiltração de diferentes materiais poliméricos e MWCO (de 0,15 a $150 \mathrm{kDa}$ ) na concentração de antocianinas de extratos de Hibiscus sabdariffa L. e observou que as retenções totais de antocianinas variaram de $24 \%$, para uma membrana de $150 \mathrm{kDa}$ a um valor de pressão transmembrana de 5 bar, a $97 \%$ para uma membrana de $5 \mathrm{kDa}$ a 30 bar (Cissé, Vaillant, Pallet, \& Dornier, 2018).

Em relação a cor notou-se que o valor de L (luminosidade) tendendo de 0 para escuro a 100 para branco variaram com o processo de filtração por membrana. O valor de L inicial do extrato da fruta da alimentação foi 85,9. Após os processos filtração na membrana de ultrafiltração de $150 \mathrm{kDa}$ o valor de L para o permeado diminuiu para 75,6, respectivamente. Os valores de $\mathrm{a}^{*}$ e b* também diminuíram após os processos de filtração nas membranas de $50 \mathrm{kDa}$ e $150 \mathrm{kDa}$. Estudo anterior que avaliou a aplicação do processo de filtração através de membrana assimétrica de fibra oca com poros variando de 0,16 a 5,29 $\mu \mathrm{m}$ para a clarificação do extrato de hibisco também observou alterações na luminosidade do permeado, sendo que o valor do extrato inicial foi de 15,69 e aumento para 22,03 para o permeado (Paraíso et al., 2020).

Em relação ao teor de sólidos totais, observou-se que após o processo de filtração por membrana assistido por ultrassom diminuiu em $20 \%$ e $7 \%$, para as membranas de $50 \mathrm{kDa}$ e $150 \mathrm{kDa}$, respectivamente. Estudo recente avaliou o processo de filtração por membrana sequencial e direta para a clarificação e concentração de compostos fenólico do extrato do fruto do pequi, e observou que o processo sequencial de microfiltração $(0,22 \mu \mathrm{m})$ e ultrafiltração $(5 \mathrm{kDa})$ reduziu 
significativamente a turbidez e o teor de sólidos do extrato (de Santana Magalhães, de Souza Martins Sá, Luiz Cardoso, \& Hespanhol Miranda Reis, 2019).

Tabela 2. Concentração de compostos fenólicos específicos e características do extrato, permeado e concentrado de cajámanga com ultrassom.

\begin{tabular}{|c|c|c|c|c|c|c|}
\hline Composto & Extrato & M08 & Perm. 50kDa & Conc. 50kDa & Perm. 150kDa & Conc. $150 \mathrm{kDa}$ \\
\hline $\begin{array}{l}\text { Ácido gálico } \\
(\mathrm{mg} / \mathrm{mL})\end{array}$ & $31,27^{\mathrm{a}} \pm 0,02$ & $90,52^{\mathrm{d}} \pm 0,03$ & $76,39^{\mathrm{c}} \pm 0,01$ & $86,52^{\mathrm{d}} \pm 0,01$ & $52,38^{b} \pm 0,02$ & $57,91^{\mathrm{b}} \pm 0,06$ \\
\hline $\begin{array}{c}\text { Ácido caféico } \\
(\mathrm{mg} / \mathrm{mL})\end{array}$ & $20,84^{\mathrm{a}} \pm 0,01$ & $67,02^{\mathrm{d}} \pm 0,01$ & $50,93^{\mathrm{c}} \pm 0,02$ & $57,68^{c} \pm 0,01$ & $34,92^{\mathrm{b}} \pm 0,05$ & $38,61^{\mathrm{b}} \pm 0,02$ \\
\hline $\begin{array}{l}\text { Ácido clorogênico } \\
(\mathrm{mg} / \mathrm{mL})\end{array}$ & $34,49^{b} \pm 0,02$ & $35,52^{\mathrm{b}} \pm 0,01$ & $40,37^{\mathrm{bc}} \pm 0,01$ & $35,83^{\mathrm{b}} \pm 0,02$ & $5,23^{\mathrm{a}} \pm 0,06$ & $7,02^{\mathrm{a}} \pm 0,01$ \\
\hline $\begin{array}{l}\text { Rutina } \\
(\mathrm{mg} / \mathrm{mL})\end{array}$ & $7,38^{\mathrm{b}} \pm 0,03$ & $9,82^{\mathrm{ab}} \pm 0,02$ & $6,79^{\mathrm{a}} \pm 0,01$ & $7,54^{\mathrm{b}} \pm 0,03$ & $5,36^{\mathrm{c}} \pm 0,03$ & $11,49^{\mathrm{a}} \pm 0,03$ \\
\hline $\begin{array}{l}\text { Quercitina } \\
\text { (mg/mL) }\end{array}$ & $25,25^{\mathrm{a}} \pm 0,02$ & $28,87^{\mathrm{ab}} \pm 0,06$ & $23,91^{\mathrm{a}} \pm 0,04$ & $21,53^{\mathrm{a}} \pm 0,22$ & $40,12^{\mathrm{c}} \pm 0,06$ & $30,17^{\mathrm{b}} \pm 0,09$ \\
\hline $\begin{array}{c}\text { Turbidez } \\
\text { (NTU) }\end{array}$ & $121,0^{\mathrm{d}} \pm 0,2$ & $9,2^{\mathrm{ab}} \pm 0,65$ & $5,0^{\mathrm{a}} \pm 0,34$ & $84,3^{c} \pm 0,33$ & $12,7^{b} \pm 0,46$ & $132,5^{\mathrm{d}} \pm 0,01$ \\
\hline $\begin{array}{l}\text { Sólidos totais } \\
\quad(\mathrm{mg} / \mathrm{L})\end{array}$ & $40060^{\mathrm{d}} \pm 0,11$ & $31060^{\mathrm{b}} \pm 0,47$ & $24840^{\mathrm{a}} \pm 0,61$ & $33220^{\mathrm{b}} \pm 0,99$ & $28680^{\mathrm{ab}} \pm 0,78$ & $35920^{\mathrm{c}} \pm 0,77$ \\
\hline $\begin{array}{c}\text { TCP } \\
(\mathrm{mg} \mathrm{EAG/g)}\end{array}$ & $1,43^{\mathrm{c}} \pm 0,55$ & $1,24^{\mathrm{c}} \pm 0,89$ & $0,45^{\mathrm{a}} \pm 0,15$ & $1,84^{\mathrm{c}} \pm 0,74$ & $0,84^{\mathrm{b}} \pm 0,06$ & $1,20^{\mathrm{bc}} \pm 0,77$ \\
\hline $\mathrm{L}$ & $65,7^{\mathrm{a}} \pm 0,01$ & $85,9^{c} \pm 0,06$ & $84,0^{c} \pm 0,04$ & $80,0^{c} \pm 0,03$ & $75,6^{\mathrm{b}} \pm 0,05$ & $76,5^{\mathrm{b}} \pm 0,04$ \\
\hline$a^{*}$ & $-4,6^{c} \pm 0,03$ & $-4,6^{c} \pm 0,03$ & $-4,2^{\mathrm{b}} \pm 0,01$ & $-4,4^{\mathrm{b}} \pm 0,02$ & $-3,8^{a} \pm 0,03$ & $-3,8^{a} \pm 0,07$ \\
\hline$b^{*}$ & $15,5^{\mathrm{c}} \pm 0,06$ & $5,4^{\mathrm{b}} \pm 0,02$ & $3,8^{a} \pm 0,01$ & $5,1^{\mathrm{b}} \pm 0,01$ & $3,8^{\mathrm{a}} \pm 0,01$ & $4,9^{\mathrm{b}} \pm 0,02$ \\
\hline
\end{tabular}

Nota. TCP-Teor de Compostos Fenólicos. Letras iguais na mesma linha indicam diferença significativa ( $\mathrm{p} \leq 0,05)$. Fonte: Autores.

\section{Conclusão}

A membrana de microfiltração avaliada $(0,8 \mu \mathrm{m})$ foi eficaz na clarificação e retenção de sólidos do extrato inicial podendo ser utilizada como um pré-tratamento. O processo de ultrafiltração assistido por ultrassom foi eficiente no aumento de fluxo de permeado, sendo que ocorreu um aumento de 64\% para a membrana de $50 \mathrm{kDa}$ e $16 \%$ para a de $150 \mathrm{kDa}$.

Assim, o processo sequencial de pré-tratamento (microfiltração) e filtração através de membranas de ultrafiltração assistido por ultrassom pode ser potencialmente aplicado para produzir extratos clarificados de cajá-manga, com teores significativos de compostos bioativos. São sugeridos estudos posteriores para avaliar outros métodos para o aumento do fluxo do permeado durante a filtração do extrato de cajá-manga e uma possível aplicação do extrato clarificado em matriz alimentícia.

\section{Referências}

AOAC. (2016). Official Methods of Analysis, (20th ed.) Association of Official Analytical Chemists.

Bindes, M. M. M., Cardoso, V. L., Reis, M. H. M., \& Boffito, D. C. (2019). Maximisation of the polyphenols extraction yield from green tea leaves and sequential clarification. Journal of Food Engineering, 241, 97-104. https://doi.org/10.1016/j.jfoodeng.2018.08.006

Breda, C. A., Justi, P. N., \& Sanjinez-argandoña, E. J. (2013). Efeito Da Desidratação Foam Mat Na Retenção Da Vitamina C Da Polpa De Cajamanga. Limentos e Nutrição = Brazilian Journal of Food and Nutrition, 24(3), 189-193.

Cassano, A., De Luca, G., Conidi, C., \& Drioli, E. (2017). Effect of polyphenols-membrane interactions on the performance of membrane-based processes. A review. Coordination Chemistry Reviews, 351, 45-75. https://doi.org/10.1016/j.ccr.2017.06.013 
Cissé, M., Vaillant, F., Pallet, D., \& Dornier, M. (2018). Selecting ultrafiltration and nanofiltration membranes to concentrate anthocyanins from roselle extract (Hibiscus sabdariffa L.). Food Research International, 44(9), 2607-2614. https://doi.org/10.1016/j.foodres.2011.04.046

Córdova, A., Astudillo-Castro, C., Ruby-Figueroa, R., Valencia, P., \& Soto, C. (2020). Recent advances and perspectives of ultrasound assisted membrane food processing. Food Research International, 133, 109163. https://doi.org/10.1016/j.foodres.2020.109163

de Santana Magalhães, F., de Souza Martins Sá, M., Luiz Cardoso, V., \& Hespanhol Miranda Reis, M. (2019). Recovery of phenolic compounds from pequi (Caryocar brasiliense Camb.) fruit extract by membrane filtrations: Comparison of direct and sequential processes. Journal of Food Engineering, 257(March), 26-33. https://doi.org/10.1016/j.jfoodeng.2019.03.025

dos Santos, S. S., Rodrigues, L. M., Cardoso, V. L., Reis, M. H. M., \& Madrona, G. S. (2018). Microcapsules of Cajá-manga (Spondias dulcis Parkinson): Influence of Different Types of Encapsulating Agents and Drying Technology. Current Nutrition \& Food Science, 15(6), 557-564. https://doi.org/10.2174/1573401314666180509144542

Hou, D., Wang, Z., Li, G., Fan, H., Wang, J., \& Huang, H. (2015). Ultrasonic assisted direct contact membrane distillation hybrid process for membrane scaling mitigation. Desalination, 375, 33-39. https://doi.org/10.1016/j.desal.2015.07.018

Lago-Vanzela, E. S., Ramin, P., Umsza-Guez, M. A., Santos, G. V., Gomes, E., \& Silva, R. da. (2011). Chemical and sensory characteristics of pulp and peel "cajá-manga" (Spondias cytherea Sonn.) jelly. Ciência e Tecnologia de Alimentos, 31(2), 398-405. https://doi.org/10.1590/s0101-20612011000200018

Louback, E., Pereira, T. A. R., De Souza, S. R., De Oliveira, J. A., \& Da Silva, L. C. (2016). Vegetation damage in the vicinity of an aluminum smelter in Brazil. Ecological Indicators, 67, 193-203. https://doi.org/10.1016/j.ecolind.2016.02.044

Machado, M. T. C., Mello, B. C. B. S., \& Hubinger, M. D. (2015). Evaluation of pequi (Caryocar Brasiliense Camb.) aqueous extract quality processed by membranes. Food and Bioproducts Processing, 95, 304-312. https://doi.org/10.1016/j.fbp.2014.10.013

Madrona, G. S., Terra, N. M., Filho, U. C., Santana, F. De, Cardoso, V. L., \& Reis, M. H. M. (2018). Purification of phenolic compounds from genipap ( Genipa americana L .) extract by the ultrasound assisted ultrafiltration process. Acta Scientiarum, 41, 1-10. https://doi.org/10.4025/actascitechnol.v41i1.35571

Meija, J. A. A., Parpinello, G. P., Versari, A., Conidi, C., \& Cassano, A. (2019). Microwave-assisted extraction and membrane-based separation of biophenols from red wine lees. Food and Bioproducts Processing, 117, 74-83. https://doi.org/10.1016/j.fbp.2019.06.020

Paraíso, C. M., dos Santos, S. S., Pereira Bessa, L., Lopes, A. P., Ogawa, C. Y. L., da Costa, S. C., \& Madrona, G. S. (2020). Performance of asymmetric spinel hollow fiber membranes for hibiscus (Hibiscus sabdariffa L.) extract clarification: Flux modeling and extract stability. Journal of Food Processing and Preservation, 44(12), 1-13. https://doi.org/10.1111/jfpp.14948

Pereira, A. S., Shitsuka, D., Parreira, F. J., \& Shitsuka, R. (2018). Metodologia da Pesquisa Científica. UFSM. https://repositorio.ufsm.br/bits tream/handle/1/15824/Lic_Computacao_Metodologia-Pesquisa-Cientifica.pdf?sequence=1

Rao, L., Hayat, K., Lv, Y., Karangwa, E., Xia, S., Jia, C., \& Zhang, X. (2011). Effect of ultrafiltration and fining adsorbents on the clarification of green tea. Journal of Food Engineering, 102(4), 321-326. https://doi.org/10.1016/j.jfoodeng.2010.09.007

Ribeiro, L. F., Ribani, R. H., Francisco, T. M. G., Soares, A. A., Pontarolo, R., \& Haminiuk, C. W. I. (2015). Profile of bioactive compounds from grape pomace (Vitis vinifera and Vitis labrusca) by spectrophotometric, chromatographic and spectral analyses. Journal of Chromatography B: Analytical Technologies in the Biomedical and Life Sciences, 1007, 72-80. https://doi.org/10.1016/j.jchromb.2015.11.005

Rodrigues, L. M., Romanini, E. B., Silva, E., Pilau, E. J., da Costa, S. C., \& Madrona, G. S. (2020). Camu-camu bioactive compounds extraction by ecofriendly sequential processes (ultrasound assisted extraction and reverse osmosis). Ultrasonics Sonochemistry, 64(January), 105017. https://doi.org/10.1016/j.ultsonch.2020.105017

Silva, F. C., Rossi, D. A., Cardoso, V. L., \& Reis, M. H. M. (2016). Suco de açaí estabilizado utilizando processo de microfiltração. Acta Scientiarum Technology, 38(1), 7-11. https://doi.org/10.4025/actascitechnol.v38i1.27037

Singleton, V. L., \& Rossi, J. A. (1965). Colorimetry of Total Phenolics with Phosphomolybdic-Phosphotungstic Acid Reagents. American Journal of Enology and Viticulture, 16(3), 144-158.

Sousa, L. dos S., Cabral, B. V., Madrona, G. S., Cardoso, V. L., \& Reis, M. H. M. (2016). Purification of polyphenols from green tea leaves by ultrasound assisted ultrafiltration process. Separation and Purification Technology, 168, 188-198. https://doi.org/10.1016/j.seppur.2016.05.029

Terra, N. M., Madrona, G. S., Ferreira, F. B., Cardoso, V. L., \& Reis, M. H. M. (2019). High Performance of Asymmetric Alumina Hollow Fiber Membranes for the Clarification of Genipap (Genipa americana L.) Fruit Extract. Food and Bioprocess Technology, 12(1), 27-38. https://doi.org/10.1007/s11947-018$2185-3$

Tylkowski, B., Trusheva, B., Bankova, V., Giamberini, M., Peev, G., \& Nikolova, A. (2010). Extraction of biologically active compounds from propolis and concentration of extract by nanofiltration. Journal of Membrane Science, 348(1-2), 124-130. https://doi.org/10.1016/j.memsci.2009.10.049 